\title{
Evaluación del proceso de producción de detergente en polvo a partir de la simulación
}

\author{
Junior Lorenzo Llanes* \\ Lourdes Zumalacárregui de Cárdenas \\ Osney Pérez Ones ${ }^{* * *}$
}

Recibido: 13/01/2015 - Aceptado: 11/12/2015

DOI: $10.22395 /$ rium.v15n28a12

\begin{abstract}
Resumen
En Cuba la producción de detergente en polvo se lleva a cabo por una sola fábrica. El presente trabajo tuvo como objetivo evaluar el proceso de producción de detergente en polvo a partir de la simulación. Para ello se empleó el simulador de procesos químicos HYSYS versión 3.2, siendo necesaria la determinación de propiedades de componentes hipotéticos que no forman parte de la base de datos de componentes puros. Los resultados del caso base mostraron ineficiencias en la operación del proceso, las más importantes, en la etapa de neutralización y en el calentamiento de los gases de secado. Los resultados del caso estudio 2 muestran que es posible reducir el $\mathrm{pH}$ de la pasta neutra en un 15,34 \% respecto al caso base, para una producción de $848 \mathrm{~kg}$ pasta neutra $/ \mathrm{h}$, sin afectar el porcentaje de ingrediente activo. Por otra parte, una reducción en el consumo de gas licuado de $1.500 \mathrm{~L} / \mathrm{d}$ implica un aumento de las ganancias por valor de 227.301 \$año con respecto al caso base.
\end{abstract}

Palabras clave: producción de detergente, simulación, HYSYS

* MSc, Profesor Asistente, Miembro del Grupo de Análisis de Procesos, Facultad de Ingeniería Química, Universidad Tecnológica de La Habana José Antonio Echeverría, Cujae. Calle 114, No. 11901, e/ Ciclovía y Rotonda, Marianao, La Habana, Cuba, e-mail: junior@quimica.cujae.edu.cu

** Ph. D., Profesora Titular, Consultante y de Mérito, Jefe del Grupo de Análisis de Procesos, Facultad de Ingeniería Química, Universidad Tecnológica de La Habana José Antonio Echeverría, Cujae. Calle 114, No. 11901, e/ Ciclovía y Rotonda, Marianao, La Habana, Cuba, e-mail: lourdes@quimica.cujae.edu.cu

*** Ph. D., Profesor Auxiliar, Miembro del Grupo de Análisis de Procesos, Facultad de Ingeniería Química, Universidad Tecnológica de La Habana José Antonio Echeverría, Cujae. Calle 114, No. 11901, e/ Ciclovía y Rotonda, Marianao,La Habana, Cuba, e-mail: osney@quimica.cujae.edu.cu 


\title{
Evaluation of the powder detergent production process from simulation
}

\begin{abstract}
In Cuba, powder detergent production is carried out by a single factory. This work research was intended to evaluate the powder detergent production process from a simulation process. For this purpose, the HYSYS v. 3.2 chemical process simulator was employed and definition of properties of hypothetical components that are nor part of the pure components database was required. Results of the basic case showed inefficiencies of the process operation, the most important being during the neutralization stage and the drying gas heating stage. Results of Study Case 2 show that it is possible to reduce $\mathrm{pH}$ of the neutral paste to $15.34 \%$ when compared to the basic case, for a production of $848 \mathrm{~kg}$ neutral paste per hour, without affecting the percentage of the active ingredient. Additionally, a decreased consumption of liquefied gas of 1,500 L/d implies increased profit in the amount of $\$ 227,301$ per year, compared to the basic case.
\end{abstract}

Key words: Detergent production; simulation; HYSYS. 


\section{INTRODUCCIÓN}

En Cuba, la producción de detergente en polvo se lleva a cabo por una sola fábrica. La misma se enfrenta a la necesidad de elevar su eficiencia y eficacia con el objetivo de aplicar los lineamientos de la política económica y social del Partido y la Revolución que le permitan insertarse exitosamente en el nuevo modelo económico cubano. Actualmente, la carencia de instrumentos de medición, así como de sistemas automatizados de control en dicha entidad impide una adecuada evaluación del mismo.

Lo anterior repercute de forma negativa en la toma de decisiones para incrementar los rendimientos. Por ello el presente trabajo tuvo como objetivo evaluar el proceso de producción de detergente en polvo a partir de la simulación. Según [1], con el propósito de entender el comportamiento de un sistema o de evaluar varias estrategias para la operación del mismo, se acude a la simulación.

El simulador de procesos HYSYS versión 3.2 es uno de los simuladores más respetados en la industria. El mismo integra la simulación en estado estacionario y la simulación dinámica [2].

El proceso de producción de detergente en polvo consta de tres etapas fundamentales: neutralización, en la cual se neutraliza el ácido dodecil-benceno sulfónico lineal (ADBS) con hidróxido de sodio y se añade agua, obteniéndose una pasta neutra cuyo ingrediente activo es el dodecil-benceno sulfonato de sodio (LAS), que pasa a un tanque de maduración para la culminación de la reacción. Este tanque está conectado a una bomba de recirculación que mantiene la pasta en movimiento.

En este paso se añade agua, la cual debe ser controlada para no afectar la humedad de la pasta neutra [3].

Si la pasta se considera conforme, según las especificaciones del proceso, pasa a la siguiente etapa, la batición. Durante la batición se mezcla la pasta neutra con el resto de las materias primas que componen el detergente y que le dan las propiedades necesarias y mejoran su calidad. La batidora carga automáticamente los materiales, con excepción del aditivo sólido que lo añade manualmente el operador auxiliar que descarga las materias primas. Según las normas de buenas prácticas se deben tomar muestras de la pasta de batidora en el tanque de alimentación como mínimo dos veces en el turno de trabajo y enviarlas al laboratorio para determinar el porcentaje de humedad cuya especificación es $28-32 \%$ [4].

La tercera etapa es el secado. En esta etapa la pasta húmeda, salida del proceso de batición, se atomiza empleando una bomba de alta presión. En estas condiciones se alimenta la torre de secado a contracorriente con aire caliente a $380^{\circ} \mathrm{C}$ aproximada- 
mente. A la torre se alimenta un volumen de aire caliente suficiente para suministrar el calor necesario para completar la evaporación del agua. La transferencia de masa y energía se logra mediante el contacto directo del gas caliente con las gotas dispersas. A la salida de la torre, el gas que sale por la parte superior se enfría (para no dañar directamente la biodiversidad) y las partículas finas se separan del gas por medio de un filtro (para que las partículas finas no pasen a la atmósfera contaminándola). La torre de aspersión generalmente está construida con chapa de acero común; está provista de mirillas o puntos de observación e inyectores de pulverización. Una vez obtenido el polvo, se le atomiza perfume y se obtiene el polvo de detergente que se usa en los hogares, hospitales, industrias, etc.

\section{MATERIALES Y MÉTODOS}

Para llevar a cabo la evaluación del proceso de producción de detergente en polvo se empleó la simulación como herramienta. Para ello se utilizó el simulador de procesos HYSYS versión 3.2, dado que el mismo dispone de rigurosos modelos para la evaluación de propiedades físicas y termodinámicas y una base de datos de cerca de 1.500 componentes que permite el cálculo de propiedades de compuestos reales e hipotéticos; cuenta con un amplio conjunto de operaciones unitarias y otros utilitarios [2].

\subsection{Simulación con HYSYS}

En la base de datos del simulador HYSYS versión 3.2 no aparecen varios de los compuestos que intervienen en el proceso; por ello fue necesario crearlos como componentes hipotéticos. Los componentes creados, así como las propiedades que se tuvieron en cuenta, se muestran en la tabla 1.

Tabla 1. Propiedades de los componentes hipotéticos

\begin{tabular}{|c|c|c|}
\hline Componentes & Propiedades & Valores \\
\hline \multirow{3}{*}{$\begin{array}{c}\text { Ácido dodecil-benceno sulfónico } \\
\mathrm{C}_{12} \mathrm{H}_{25} \mathrm{C}_{6} \mathrm{H}_{4} \mathrm{SO}_{3} \mathrm{H} \\
{[5]}\end{array}$} & Masa molar & $326 \mathrm{~kg} / \mathrm{kmol}$ \\
\cline { 2 - 3 } & Densidad & $1.050 \mathrm{~kg} / \mathrm{m}^{3}$ \\
\cline { 2 - 3 } & Punto de fusión & $284^{\circ} \mathrm{C}$ \\
\cline { 2 - 3 } & Punto de ebullición & $654^{\circ} \mathrm{C}$ \\
\cline { 2 - 3 } & Presión de vapor a $25^{\circ} \mathrm{C}$ & $6^{*} 10^{-7} \mathrm{MPa}$ \\
\hline $\begin{array}{c}\text { Dodecil-benceno sulfonato de sodio } \\
\mathrm{C}_{12} \mathrm{H}_{25} \mathrm{C}_{6} \mathrm{H}_{4} \mathrm{SO}_{3} \mathrm{Na} \\
{[6]}\end{array}$ & Masa molar & $348 \mathrm{~kg} / \mathrm{kmol}$ \\
\cline { 2 - 3 } & Densidad & $1.071 \mathrm{~kg} / \mathrm{m}^{3}$ \\
\hline
\end{tabular}


Evaluación del proceso de producción de detergente en polvo a partir de la simulación 245

\begin{tabular}{|c|c|c|}
\hline Componentes & Propiedades & Valores \\
\hline \multirow{3}{*}{$\begin{array}{l}\text { Carbonato de sodio } \\
\qquad \mathrm{Na}_{2} \mathrm{CO}_{3}[7]\end{array}$} & Masa molar & $106 \mathrm{~kg} / \mathrm{kmol}$ \\
\hline & Calor de formación & $-1.127 .959 \mathrm{~kJ} / \mathrm{kmol}$ \\
\hline & Densidad & $2.553 \mathrm{~kg} / \mathrm{m}^{3}$ \\
\hline \multirow{2}{*}{$\begin{array}{l}\text { Sulfato de sodio } \\
\qquad \mathrm{Na}_{2} \mathrm{SO}_{4}[7]\end{array}$} & Masa molar & $142 \mathrm{~kg} / \mathrm{kmol}$ \\
\hline & Densidad & $2.698 \mathrm{~kg} / \mathrm{m}^{3}$ \\
\hline \multirow{2}{*}{$\begin{array}{l}\text { Silicato de sodio } \\
\qquad \mathrm{Na}_{2} \mathrm{SiO}_{3}[7]\end{array}$} & Masa molar & $114 \mathrm{~kg} / \mathrm{kmol}$ \\
\hline & Densidad & $1.526 \mathrm{~kg} / \mathrm{m}^{3}$ \\
\hline
\end{tabular}

Fuente: elaboración propia

Para la determinación de las capacidades calóricas del ADBS y del LAS se utilizaron dos métodos de contribución de grupos dados por [8].

Método 1: Ley de Kopp's modificada por Werner en 1941 para sólidos. En este caso las capacidades calóricas del compuesto se obtienen a partir de la sumatoria del producto del número de átomos de cada elemento presente por un factor empírico para cada elemento, el cual depende del estado de agregación del compuesto en cuestión. En la tabla 2 se muestran los resultados de la aplicación del método.

Tabla 2. Evaluación de la capacidad calórica del LAS

\begin{tabular}{|c|c|c|c|}
\hline Elemento & $N^{\circ}$ de átomos & Factor & $C p(\mathrm{~kJ} / \mathrm{kmol} \mathrm{K})$ \\
\hline $\mathrm{C}$ & 18 & 7,5 & 135,0 \\
\hline $\mathrm{H}$ & 29 & 9,6 & 278,4 \\
\hline $\mathrm{O}$ & 3 & 16,7 & 50,1 \\
\hline $\mathrm{S}$ & 1 & 22,6 & 22,6 \\
\hline $\mathrm{Na}$ & 1 & 26,0 & 26,0 \\
\hline Total & & & 512,0 \\
\hline
\end{tabular}

Fuente: elaboración propia

Método 2: contribución de grupos de Chunch y Swanson (1973) para líquidos orgánicos. En este caso la capacidad calórica del compuesto se obtiene a partir de la sumatoria de la contribución de un factor reportado para cada grupo funcional $\left(\mathrm{CH}_{3}\right.$, $\mathrm{CH}_{2}, \mathrm{CH}$ ), por el número de veces que aparece dicho grupo en la estructura. Este factor depende del estado de agregación del compuesto en cuestión. En la tabla 3 se muestran los resultados de la aplicación del método. 
Tabla 3. Evaluación de la capacidad calórica del ADBS

\begin{tabular}{|c|c|c|c|}
\hline Elemento y/o grupo & Cantidad & Factor & $C p(\mathrm{~kJ} / \mathrm{kmolK})$ \\
\hline $\mathrm{CH}_{3}$ & 2 & 36,84 & 73,68 \\
\hline $\mathrm{CH}_{2}$ & 9 & 30,40 & 273,60 \\
\hline $\mathrm{CH}$ & 1 & 20,93 & 20,93 \\
\hline$-\mathrm{C} H=$ & 4 & 18,42 & 73,68 \\
\hline$-\mathrm{C}=$ & 2 & 12,14 & 24,28 \\
\hline $\mathrm{O}$ & 3 & 35,17 & 105,51 \\
\hline $\mathrm{H}$ & 1 & 14,65 & 14,65 \\
\hline Total & & & 586,33 \\
\hline
\end{tabular}

Fuente: elaboración propia

La búsqueda de buenos valores para propiedades físicas de los parámetros es la clave para una simulación exitosa. Lo anterior depende mucho de escoger el método correcto de estimación de propiedades. Los cuatro factores que se deben tener en cuenta al escoger los métodos de estimación de propiedades son: la naturaleza de las propiedades de interés; la composición de la mezcla; los intervalos de presión y temperaturas, y la disponibilidad de parámetros.

Dada la presencia de electrolitos en el proceso de producción de detergentes se hace necesaria la selección de un paquete de propiedades que se ajuste a esta situación. Los métodos más comunes para el trabajo con electrolitos son el Pitzer y el NRTL-modificado [9]. En el presente trabajo, teniendo en cuenta la presencia de compuestos orgánicos y agua, se empleó el paquete NRTL-modificado, ya que la ecuación de Pitzer no considera la interacción de las partículas con las moléculas de solvente [10].

En la tabla 4 se muestran los módulos empleados para simular las diferentes operaciones unitarias presentes en el proceso, así como los equipos asociados a estas. 
Tabla 4. Módulos del simulador HYSYS y operaciones unitarias asociadas

\begin{tabular}{|c|c|c|}
\hline Operación unitaria & Módulo & Equipos \\
\hline Reacción química & $\begin{array}{l}\text { Reactor de conversión } \\
\text { (conversion reactor) }\end{array}$ & $\begin{array}{l}\text { - Neutralizador } \\
\text { - Tanque de maduración } \\
\text { - Horno }\end{array}$ \\
\hline Flujo de fluidos & Tanque (tank) & - Batidora \\
\hline & $\overbrace{\text { Bomba (pump) }}$ & - Bombas \\
\hline \multirow[t]{2}{*}{$\begin{array}{l}\text { Transferencia de calor } \\
\text { Transferencia de masa }\end{array}$} & Calentador (heater) & \multirow[t]{2}{*}{ - Torre de secado } \\
\hline & Divisor de flujo (splitter) & \\
\hline
\end{tabular}

Fuente: elaboración propia

A continuación se describen los parámetros que se fijaron para la simulación de cada una de las operaciones unitarias. 


\subsubsection{Neutralización}

Para la simulación de esta operación se crearon tres corrientes de entrada con sus correspondientes variables de decisión, todas a $25^{\circ} \mathrm{C}$ y $101,3 \mathrm{kPa}$, que corresponden a los reactivos (ADBS y $\mathrm{NaOH}(\mathrm{ac})$ ), así como una corriente de agua. Las especificaciones se muestran en la tabla 5. Por otra parte se creó una corriente de salida (LAS) con una temperatura de $55^{\circ} \mathrm{C}$ y presión de $101,3 \mathrm{kPa}$. También fue añadida una corriente de energía (Qperdido) de manera que el simulador determinase la energía cedida en el proceso. La corriente de salida del neutralizador constituye la entrada a un segundo reactor de conversión el cual es el equivalente al tanque intermedio descrito en el proceso. En este caso solo se fijó la presión a la corriente de salida.

Tabla 5. Corrientes de entrada al neutralizador

\begin{tabular}{|c|c|c|}
\hline Corriente & Flujo $(\mathrm{kg} / \mathrm{h})$ & Composición (\% másico) \\
\hline ADBS & 333 & 1,0 \\
\hline $\mathrm{NaOH}_{(\mathrm{ac})}$ & 92,91 & 0,5 \\
\hline $\mathrm{H}_{2} \mathrm{O}$ & 459 & 1,0 \\
\hline
\end{tabular}

Fuente: elaboración propia

Como uno de los parámetros a controlar en la etapa es el $\mathrm{pH}$, fue necesario crear una hoja de cálculo (spreadsheet) para conocer su valor. El cálculo se realizó a partir de la diferencia entre la constante de disociación del agua, cuyo valor se tomó a la temperatura de $60^{\circ} \mathrm{C}$ que, según [11], tiene un valor de $9,6 \times 10^{-14}$ y el pOH de la pasta neutra, valor que se calcula a partir de la concentración de $\mathrm{NaOH}$, dado que este es un electrolito fuerte y soluble. La concentración molar de $\mathrm{NaOH}$ se determina a partir del flujo molar de $\mathrm{NaOH}(\mathrm{n}(\mathrm{NaOH})$ ) y el flujo volumétrico (Vsalida) a la salida del reactor. Para ello se emplearon las ecuaciones desde la (1) hasta la (3). La ecuación 3 está afectada por el valor de la constante de disociación del agua a las condiciones de trabajo.

$$
\begin{gathered}
c(\mathrm{NaOH})=\frac{n(\mathrm{NaOH})}{V_{\text {salida }}} \\
p O H=-\log c(\mathrm{NaOH}) \\
p H=13-p O H
\end{gathered}
$$




\subsubsection{Batición}

Para la simulación de esta operación se tienen dos corrientes de entrada. Una es la salida del tanque intermedio y otra se creó teniendo en cuenta los consumos de materias primas en la etapa (aditivos). La temperatura y presión de la corriente de Aditivos es $25^{\circ} \mathrm{C}$ y $101,3 \mathrm{kPa}$, respectivamente. En la tabla 6 se muestran las especificaciones de la corriente creada.

Tabla 6. Corrientes de entrada a la batidora

\begin{tabular}{|c|c|c|}
\hline Corriente & Componente & Flujo $(\mathrm{kg} / \mathrm{h})$ \\
\hline \multirow{4}{*}{ Aditivos } & $\mathrm{Na}_{2} \mathrm{CO}_{3}$ & 93 \\
\cline { 2 - 3 } & $\mathrm{Na}_{2} \mathrm{SiO}_{2}$ & 218,4 \\
\cline { 2 - 3 } & $\mathrm{Na}_{2} \mathrm{SO}_{4}$ & 1.624 \\
\cline { 2 - 3 } & $\mathrm{H}_{2} \mathrm{O}$ & 509,6 \\
\hline
\end{tabular}

Fuente: elaboración propia

También se creó una corriente de salida (pasta batidora) a la cual se le fijó la temperatura de $45{ }^{\circ} \mathrm{C}$ valor que se obtiene de mediciones realizadas durante el proceso productivo; por otra parte, se conectó una corriente de energía (W) de manera que el simulador evaluase el trabajo entregado por el agitador para el mezclado.

\subsubsection{Horno}

Para la simulación de esta sección se crearon dos corrientes de entrada al reactor cuyas especificaciones se muestran en la tabla 7. Además de las corrientes de entrada se creó una de salida (gas secado) donde las únicas especificaciones hechas fueron las de temperatura y presión $\left(400^{\circ} \mathrm{C}\right.$ y $\left.101,3 \mathrm{kPa}\right)$. Al igual que en el resto de las operaciones descritas anteriormente se conectó al reactor una corriente de energía (Q) de manera que se calculara el calor cedido al medio.

Tabla 7. Corrientes de entrada al horno

\begin{tabular}{|c|c|c|c|c|}
\hline \multirow{2}{*}{ Corriente } & \multicolumn{4}{|c|}{ Variables de decisión } \\
\cline { 2 - 5 } & $\begin{array}{c}\text { Temperatura } \\
\left({ }^{\circ} \mathrm{C}\right)\end{array}$ & $\begin{array}{c}\text { Presión } \\
(\mathrm{kPa})\end{array}$ & Componente & $\begin{array}{c}\text { Composición } \\
(\% \text { molar })\end{array}$ \\
\hline \multirow{2}{*}{$\begin{array}{c}\text { Aire } \\
(80,95 \mathrm{kmol} / \mathrm{h})\end{array}$} & \multirow{2}{*}{25} & \multirow{2}{*}{101,3} & $\mathrm{O}_{2}$ & 0,20 \\
\hline \begin{tabular}{c}
$\mathrm{Gas}$ \\
\cline { 3 - 5 }
\end{tabular} & \multirow{2}{*}{25} & \multirow{2}{*}{101,3} & $\mathrm{~N}_{2}$ & 0,76 \\
\hline & & $\mathrm{H}_{2} \mathrm{O}$ & 0,04 \\
\hline
\end{tabular}

Fuente: elaboración propia 


\subsubsection{Secado}

En el proceso de secado están presentes las operaciones de transferencia de calor y masa. Para la simulación de la operación de intercambio de calor a partir del uso del módulo intercambiador de calor se crearon dos corrientes de materiales a la salida, pasta a secador y aire a secador, de las cuales solo a la corriente pasta a secador se le especificó la temperatura $\left(90^{\circ} \mathrm{C}\right)$. Las corrientes de entrada fueron calculadas por el módulo que representa el horno y la bomba de alta.

Por otra parte, se consideró que no existía caída de presión ni por el lado de los tubos ni de la coraza. En el caso de la operación de transferencia de masa se empleó el módulo divisor de flujo, considerando la operación adiabática. Para ello se crearon dos corrientes de materiales (detergente y emisión) a la salida, así como una de energía $\left(Q_{1}\right)$. Para determinar las composiciones de las corrientes de salida se tuvo en cuenta que la humedad del producto final debía ser de 2,5 \% como máximo.

\subsubsection{Bombeo}

El sistema de bombeo está formado por dos bombas (alta y baja); para la simulación se tomó el módulo bomba. A cada uno de los módulos se le conectó una corriente de energía, potencia 1 y potencia 2 , respectivamente, de manera que el simulador evaluase la potencia consumida. En la figura 1 se muestra el esquema de simulación del proceso de producción de detergente en polvo.

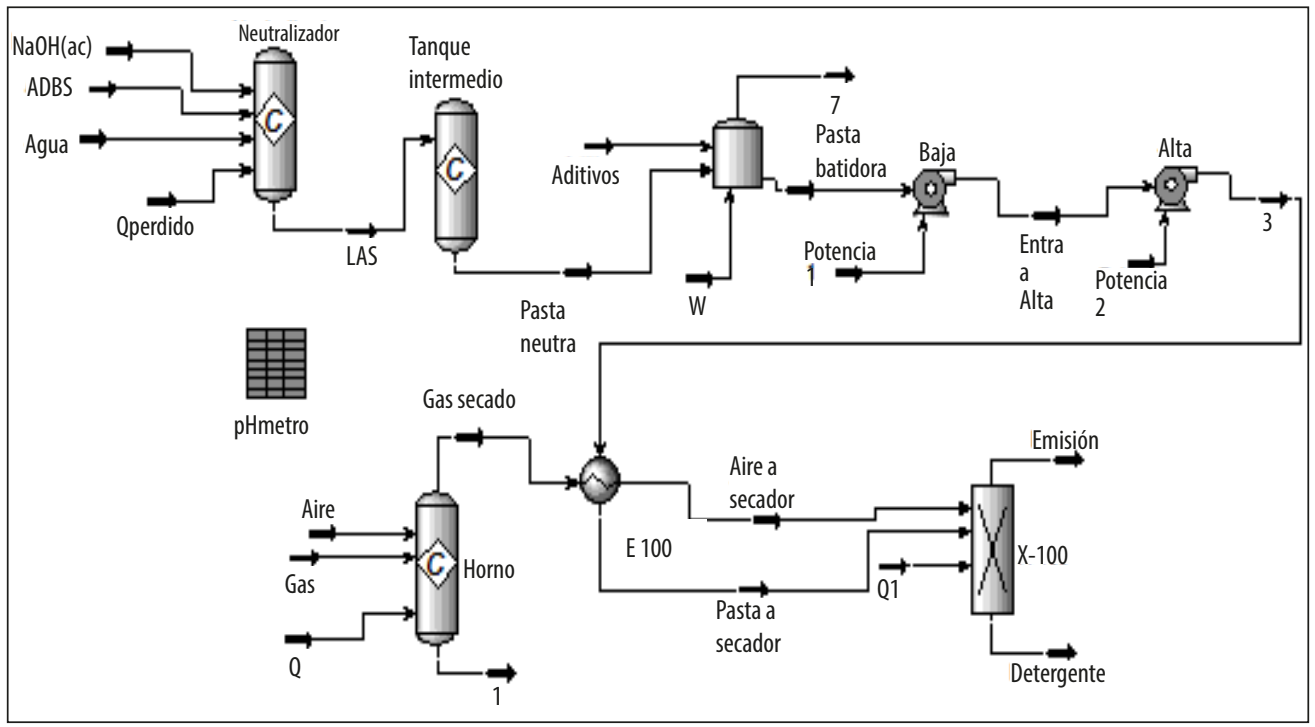

Figura 1. Esquema de simulación del proceso de producción de detergente en polvo Fuente: elaboración propia 


\section{RESULTADOS DEL CASO BASE (CB)}

\subsection{Balance de materiales y energía en la sección de neutralización}

En la tabla 8 se muestran los resultados del balance de materiales en el neutralizador y el cálculo del $\mathrm{pH}$ obtenido manualmente y a partir del simulador. Los cálculos manuales se realizaron con el objetivo de validar el modelo de simulación.

Tabla 8. Resultados del balance de materiales en el neutralizador

\begin{tabular}{|c|c|c|c|c|}
\hline \multirow{2}{*}{ Componentes } & \multicolumn{2}{|c|}{ Manual } & \multicolumn{2}{|c|}{ HYSYS } \\
\hline & Entra (kmol) & Sale (kmol) & Entra (kmol) & Sale (kmol) \\
\hline ADBS & 1,021 & 0,021 & 1,021 & 0 \\
\hline $\mathrm{NaOH}$ & 1,1614 & 0,1614 & 1,1614 & 0,1399 \\
\hline $\mathrm{H}_{2} \mathrm{O}$ & 28,08 & 29,08 & 28,08 & 29,13 \\
\hline LAS & 0 & 1,000 & 0 & 1,02 \\
\hline Pasta neutra $(\mathrm{kg})$ & - & 884 & - & 885 \\
\hline \multicolumn{4}{|c|}{ Error relativo } & $<5 \%$ \\
\hline pH pasta neutra & \multicolumn{2}{|c|}{12,11} & - & 12,15 \\
\hline \multicolumn{4}{|c|}{ Error relativo } & $<5 \%$ \\
\hline
\end{tabular}

Fuente: elaboración propia

De este resultado se aprecia que el esquema de simulación reproduce con menos del $5 \%$ de error relativo el balance de materiales en el neutralizador.

Para el cálculo de las pérdidas de energía en el neutralizador se estimó la energía cedida por convección y radiación (Qconv, Qrad). Estos valores se muestran en la tabla 9. No fue posible verificar el resultado de los balances de energía con el simulador, por no haber posibilidades de realizar mediciones experimentales que permitieran corroborar los estimados de propiedades energéticas de los componentes hipotéticos.

Tabla 9. Pérdidas de calor por convección-radiación en el neutralizador

\begin{tabular}{|c|c|c|}
\hline Parámetro & Área $\left(\mathrm{m}^{2}\right)$ & Q conv-rad $(\mathrm{kJ} / \mathrm{h})$ \\
\hline Cuerpo & 5,89 & 7.510 \\
\hline \multirow{2}{*}{ Tapa } & \multirow{2}{*}{1,33} & Q conv: 1.092 \\
\cline { 3 - 3 } & & Q rad: 138 \\
\hline Fondo & 1,24 & $\Sigma=1.230$ \\
\hline Total & 8,46 & 1.450 \\
\hline
\end{tabular}

Fuente: elaboración propia 


\subsection{Balance de energía en la sección de batición}

El resultado más importante de la etapa de batición es que la potencia requerida para realizar el mezclado de los componentes, en las condiciones de operación reportadas por la fábrica, a partir de HYSYS, es de 47,11 kW. Dado que la potencia máxima del motor del agitador es de $55 \mathrm{~kW}$, operando al $86 \%$ de eficiencia puede suministrar el valor requerido.

\subsection{Balance de materiales y energía en el horno}

En la tabla 10 se presenta el resultado del balance de materiales en el horno.

De este resultado se aprecia que el esquema de simulación reproduce con menos del $5 \%$ de error el balance de materiales en el horno.

Para el balance de energía, el cálculo del calor cedido al medio durante la combustión del gas en el horno es $4,46 \times 10^{6} \mathrm{~kJ} / \mathrm{h}$, que representa que el $80 \%$ está siendo cedido al medio lo que evidencia la baja eficiencia del sistema. Una manifestación de esta ineficiencia en el intercambio de calor en el horno es la temperatura de salida de los gases de combustión, muy por debajo de la media para una reacción de combustión que se desarrolle correctamente.

Tabla 10. Resultados del balance de materiales en el horno

\begin{tabular}{|c|c|c|c|c|}
\hline \multirow{2}{*}{ Componentes } & \multicolumn{2}{|c|}{ Manual } & \multicolumn{2}{c|}{ HYSYS } \\
\cline { 2 - 5 } & $\begin{array}{c}\text { Entra } \\
(\mathrm{kmol})\end{array}$ & $\begin{array}{c}\text { Sale } \\
(\mathrm{kmol})\end{array}$ & $\begin{array}{c}\text { Entra } \\
(\mathrm{kmol})\end{array}$ & $\begin{array}{c}\text { Sale } \\
(\mathrm{kmol})\end{array}$ \\
\hline $\mathrm{C}_{3} \mathrm{H}_{8}$ & 0,926 & 0 & 0,9259 & 0 \\
\hline $\mathrm{C}_{4} \mathrm{H}_{10}$ & 1,389 & 0 & 1,3889 & 0 \\
\hline $\mathrm{O}_{2}$ & 16,39 & 2,73 & 16,39 & 2,73 \\
\hline $\mathrm{N}_{2}$ & 61,67 & 61,67 & 61,67 & 61,67 \\
\hline $\mathrm{H}_{2} \mathrm{O}$ & 2,89 & 13,54 & 2,89 & 13,54 \\
\hline $\mathrm{CO}_{2}$ & 0 & 8,33 & 0 & 8,33 \\
\hline \multicolumn{5}{|c}{ Error relativo } \\
\hline
\end{tabular}

Fuente: elaboración propia

\subsection{Balance de materiales y energía en la torre de secado}

Para verificar la validez de representar la torre de secado por el módulo Divisor de flujo se calculó el agua evaporada en el proceso $(973,73 \mathrm{~kg} / \mathrm{h})$ y se comparó con la 
reportada por el simulador $(976,97 \mathrm{~kg} / \mathrm{h})$, de donde el error relativo en la estimación es menor que $5 \%$.

Adicionalmente se evaluó manualmente la energía requerida para el secado y el porcentaje de pérdidas en el secador, arrojando $0,77 \times 10^{6} \mathrm{~kJ} / \mathrm{h}$ y $23,32 \%$ respectivamente.

En la tabla 11 se presenta en resultado de la evaluación del divisor de flujo según HYSYS.

Tabla 11.Resultados del divisor de flujo

\begin{tabular}{|l|c|c|c|}
\hline \multirow{2}{*}{\multicolumn{1}{|c|}{ Parámetro }} & \multicolumn{3}{c|}{ Corrientes } \\
\cline { 2 - 4 } & Emisión & Detergente & $Q_{\text {secado }}$ \\
\hline Flujo másico $(\mathrm{kg} / \mathrm{h})$ & 3390 & 2367 & - \\
\hline Flujo molar $(\mathrm{kmol} / \mathrm{h})$ & 139,8 & 19,29 & - \\
\hline Flujo volumétrico $\left(\mathrm{m}^{3} / \mathrm{h}\right)$ & 3,874 & 1,187 & - \\
\hline Entalpía molar $(\mathrm{kJ} / \mathrm{kmol})$ & $-1,363 \times 10^{5}$ & $-9,767 \times 10^{4}$ & - \\
\hline Entalpía total $(\mathrm{kJ} / \mathrm{h})$ & $-1,687 \times 10^{7}$ & $-1,884 \times 10^{6}$ & $1,948 \times 10^{6}$ \\
\hline
\end{tabular}

Fuente: elaboración propia

Si se hubiera considerado un porcentaje de pérdidas de calor en la torre de secado igual al obtenido mediante el cálculo manual, la energía requerida para el secado hubiera sido de $2,77 \times 10^{6} \mathrm{~kJ} / \mathrm{h}$, valor que difiere del calor absorbido hallado manualmente en un 9,3\%. Lo anterior indica que es posible emplear para la simulación este módulo de cálculo.

\section{DISCUSIÓN DE CASOS DE ESTUDIO (CE)}

A partir de los resultados del caso base se determinó realizar tres estudios de casos con el objetivo de valorar alternativas tecnológicas que conllevan al uso racional de materias primas y energía.

\subsection{Caso de estudio 1 (CE1)}

Los resultados del caso base muestran que el $\mathrm{pH}$ de la pasta neutra, como el porcentaje de componente activo (\% LAS) se encuentran fuera de especificaciones; de ahí que se estudiaron dos variantes tecnológicas para mejorar estos resultados. La variante tecnológica analizada consistió en disminuir el flujo de la solución de hidróxido de sodio alimentada al neutralizador hasta $82 \mathrm{~kg} / \mathrm{h}$. 
Para ello a la entrada del neutralizador se debe regular el flujo de solución de hidróxido de sodio en el cabezal de la bomba triple que corresponde a este reactivo. En la variante se analiza cómo la variable independiente (flujo másico de $\mathrm{NaOH}$ ) influye sobre las variables dependientes ( $\mathrm{pH}$ y fracción másica de componente activo). En este caso la variable independiente varía en un intervalo cuyo límite inferior es de $82 \mathrm{~kg} / \mathrm{h}$ y el superior de $92 \mathrm{~kg} / \mathrm{h}$, tomándose un paso de $1,00 \mathrm{~kg} / \mathrm{h}$. Los resultados de este caso de estudio se muestran en la figura 2.

\section{Caso de Estudio 1}

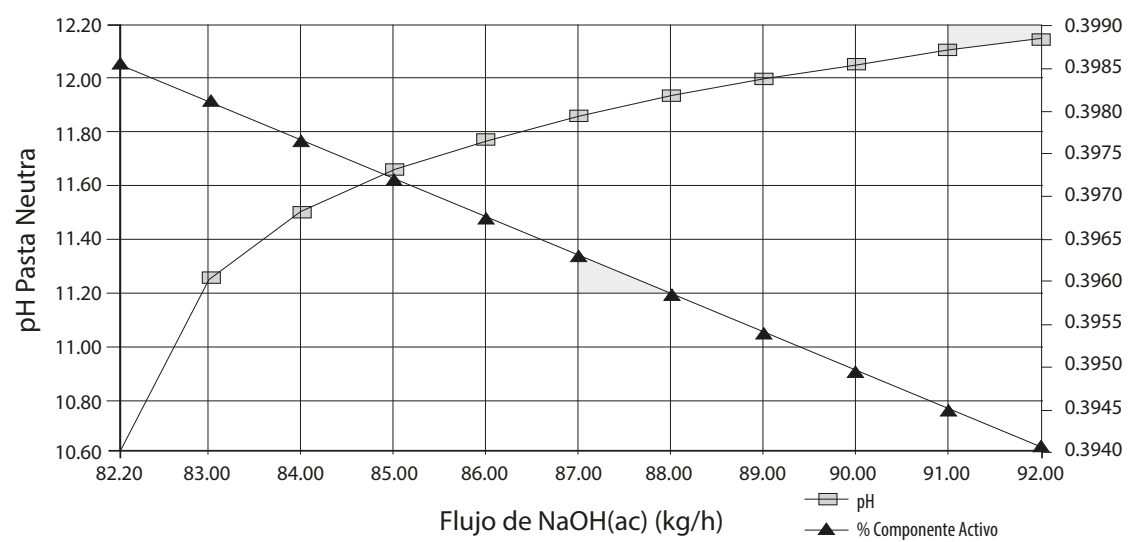

Figura 2. Dependencia del pH y la fracción másica de LAS con el consumo de $\mathrm{NaOH}(\mathrm{ac})$.

Fuente: elaboración propia

A partir del análisis de la figura 2 se puede observar que al disminuir el flujo de hidróxido de sodio desde $92 \mathrm{~kg} / \mathrm{h}$ hasta $82 \mathrm{~kg} / \mathrm{h}$ el pH de la pasta neutra disminuye desde 12,15 , valor que se encuentra fuera de especificación según la norma de neutralización [4], hasta 10,60, lo que representa una disminución en el valor del $\mathrm{pH}$ de un 15,63\%. A partir de esta modificación el exceso de hidróxido de sodio disminuye desde 13,25\% hasta $0,3 \%$. Lo anterior se debe a que al disminuir la concentración de hidróxido de sodio a la salida el valor del $\mathrm{pOH}$ aumenta lo que provoca una disminución del $\mathrm{pH}$ de la pasta neutra.

Por otra parte, se observa un aumento lineal del porcentaje de componente activo $\left(\mathrm{C}_{18} \mathrm{H}_{29} \mathrm{SO}_{3} \mathrm{Na}\right)$ desde un $39,40 \%$ hasta un $39,85 \%$, resultado que se corresponde con el balance de materiales. No obstante ambos valores se hallan fuera de especificación que según [4] debe ser (38,2\% $\% 1 \%)$. Para un flujo de $85 \mathrm{~kg} / \mathrm{h}$ de hidróxido de sodio se obtiene una mejor condición de operación, manteniendo constante el flujo de ácido al neutralizador. 


\subsection{Caso de estudio 2 (CE2)}

Al igual que en el CE1 en la variante que se analiza a continuación se pretende mejorar el resultado obtenido para el $\mathrm{pH}$ y el porcentaje de componente activo. En este caso se disminuyeron los flujos de reactivos. La variante tecnológica analizada consistió en disminuir el flujo de la solución de hidróxido de sodio hasta $75 \mathrm{~kg} / \mathrm{h}$, así como el de ADBS hasta $305 \mathrm{~kg} / \mathrm{h}$.

Para ello, a la entrada del neutralizador se deben regular los flujos de solución de hidróxido de sodio y ADBS en los cabezales de la bomba triple que corresponden a estos reactivos. En la variante se analiza cómo las variables independientes (flujo másico de $\mathrm{NaOH}(\mathrm{ac})$ y de $\mathrm{ADBS}$ ) influyen sobre las variables dependientes $\mathrm{pH}$ y fracción másica de componente activo. En este caso las variables independientes varían en los siguientes intervalos: flujo másico de $\mathrm{NaOH}$ de $70 \mathrm{~kg} / \mathrm{h}-90 \mathrm{~kg} / \mathrm{h}$ y flujo másico de ADBS entre $300 \mathrm{~kg} / \mathrm{h}-330 \mathrm{~kg} / \mathrm{h}$; para ambos el paso es 5,00 kg/h.

En este caso se produce una reducción del $\mathrm{pH}$ de la pasta neutra desde 12,25 hasta 10,37, lo que representa una reducción de un $15,34 \%$. Por otra parte, el menor flujo de ácido e hidróxido provoca una disminución del porcentaje de componente activo en la pasta neutra desde un valor de 39,23 \%, fuera de especificación, hasta 37,98 \% valor que se encuentra dentro de la norma.

Al comparar estos resultados con los del CE1 se ve que el $\mathrm{pH}$ se reduce en ambos casos prácticamente en el mismo porcentaje, aunque en el CE2 adicionalmente se disminuye el consumo de ácido. La producción de pasta neutra para las mejores condiciones del CE2 (75 kg/h de hidróxido y $305 \mathrm{~kg} / \mathrm{h}$ de ácido) es de $848 \mathrm{~kg}_{\text {pastaneutra }} / \mathrm{h}$, mientras que en las condiciones del CB es de $885 \mathrm{~kg}_{\text {pastaneutra }} / \mathrm{h}$, con una disminución de un 4,2\%. En la tabla 12 se muestra el comportamiento de las variables en el CE2.

Tabla 12. Resultados del caso de estudio 2

\begin{tabular}{|l|c|c|c|c|c|}
\hline \multicolumn{1}{|c|}{ Estado } & 1 & 2 & 3 & 4 & 5 \\
\hline ADBS- Flujo másico, $\mathrm{kg} / \mathrm{h}$ & 300,0 & 300,0 & 300,0 & 300,0 & 300,0 \\
\hline $\mathrm{NaOH}(\mathrm{ac})-$ Flujo másico, $\mathrm{kg} / \mathrm{h}$ & 70,00 & 75,00 & 80,00 & 85,00 & 90,00 \\
\hline $\mathrm{pH}$ Pasta Neutra & - & 11,31 & 1197,00 & 12,22 & 12,37 \\
\hline \% Componente Activo & 0,3673 & 0,3673 & 0,3741 & 0,3741 & 0,3697 \\
\hline
\end{tabular}


256 Junior Lorenzo Llanes - Lourdes Zumalacárregui de Cárdenas - Osney Pérez Ones

\begin{tabular}{|c|c|c|c|c|c|}
\hline Estado & 1 & 2 & 3 & 4 & 5 \\
\hline Estado & 6 & 7 & 8 & 9 & 10 \\
\hline ADBS- Flujo másico, $\mathrm{kg} / \mathrm{h}$ & 305,0 & 305,0 & 305,0 & 305,0 & 305,0 \\
\hline $\mathrm{NaOH}(\mathrm{ac})$ - Flujo másico, $\mathrm{kg} / \mathrm{h}$ & 70,00 & 75,00 & 80,00 & 85,00 & 90,00 \\
\hline pH Pasta Neutra & - & 10,37 & 11,75 & 12,11 & 12,30 \\
\hline$\%$ Componente Activo & 0,363 & 0,3842 & 0,3820 & 0,3797 & 0,3775 \\
\hline Estado & 11 & 12 & 13 & 14 & 15 \\
\hline ADBS- Flujo másico, kg/h & 310,0 & 310,0 & 310,0 & 310,0 & 310,0 \\
\hline $\mathrm{NaOH}(\mathrm{ac})-$ Flujo másico, $\mathrm{kg} / \mathrm{h}$ & 70,00 & 75,00 & 80,00 & 85,00 & 90,00 \\
\hline pH Pasta Neutra & - & - & 11,75 & 12,11 & 12,30 \\
\hline$\%$ Componente Activo & 0,363 & 0,3842 & 0,3820 & 0,3797 & 0,3775 \\
\hline Estado & 16 & 17 & 18 & 19 & 20 \\
\hline ADBS- Flujo másico, kg/h & 315,0 & 315,0 & 315,0 & 315,0 & 315,0 \\
\hline $\mathrm{NaOH}(\mathrm{ac})-$ Flujo másico, $\mathrm{kg} / \mathrm{h}$ & 70,00 & 75,00 & 80,00 & 85,00 & 90,00 \\
\hline pH Pasta Neutra & - & - & 11,59 & 12,04 & 12,26 \\
\hline$\%$ Componente Activo & 0,3608 & 0,3843 & 0,3859 & 0,3836 & 0,3814 \\
\hline Estado & 21 & 22 & 23 & 24 & 25 \\
\hline ADBS- Flujo másico, kg/h & 320,0 & 320,0 & 320,0 & 320,0 & 320,0 \\
\hline $\mathrm{NaOH}(\mathrm{ac})$ - Flujo másico, $\mathrm{kg} / \mathrm{h}$ & 70,00 & 75,00 & 80,00 & 85,00 & 90,00 \\
\hline pH Pasta Neutra & - & - & 11,32 & 11,96 & 12,21 \\
\hline$\%$ Componente Activo & 0,3587 & 0,382 & 0,3897 & 0,3874 & 0,3852 \\
\hline Estado & 26 & 27 & 28 & 29 & 30 \\
\hline ADBS- Flujo másico, kg/h & 325,0 & 325,0 & 325,0 & 325,0 & 325,0 \\
\hline $\mathrm{NaOH}(\mathrm{ac})-$ Flujo másico, $\mathrm{kg} / \mathrm{h}$ & 70,00 & 75,00 & 80,00 & 85,00 & 90,00 \\
\hline pH Pasta Neutra & - & - & 10,55 & 11,87 & 12,16 \\
\hline$\%$ Componente Activo & 0,3566 & 0,3798 & 0,3995 & 0,3912 & 0,389 \\
\hline
\end{tabular}


Evaluación del proceso de producción de detergente en polvo a partir de la simulación 257

\begin{tabular}{|l|c|c|c|c|c|}
\hline \multicolumn{1}{|c|}{ Estado } & 1 & 2 & 3 & 4 & 5 \\
\hline Estado & $\mathbf{3 1}$ & $\mathbf{3 2}$ & $\mathbf{3 3}$ & $\mathbf{3 4}$ & $\mathbf{3 5}$ \\
\hline ADBS- Flujo másico, $\mathrm{kg} / \mathrm{h}$ & 330,0 & 330,0 & 330,0 & 330,0 & 330,0 \\
\hline NaOH(ac)- Flujo másico, $\mathrm{kg} / \mathrm{h}$ & 70,00 & 75,00 & 80,00 & 85,00 & 90,00 \\
\hline pH Pasta Neutra & - & - & - & 11,75 & 12,1 \\
\hline \% Componente Activo & 0,3545 & 0,3776 & 0,3973 & 0,35 & 0,3927 \\
\hline
\end{tabular}

Fuente: elaboración propia

\subsection{Caso de estudio 3 (CE3)}

El elevado valor de las pérdidas energéticas en el horno hace necesario el estudio de alguna alternativa que permita disminuir este valor.

Variante tecnológica analizada: disminuir el flujo de gas licuado para la combustión en el horno hasta $2.000 \mathrm{~L} / \mathrm{d}$.

En este caso se analiza la influencia de la variable independiente flujo de gas licuado sobre la variable dependiente calor perdido $(\mathrm{Qp} \mathrm{H})$. Al igual que en el caso base se mantiene constante la temperatura de salida de los gases de combustión. Para la simulación de este caso de estudio se varía el valor de la variable independiente desde los $0,095 \mathrm{kmol} / \mathrm{h}$ hasta los $0,22 \mathrm{kmol} / \mathrm{h}$, tomándose un paso de $0,01 \mathrm{kmol} / \mathrm{h}$.

Una disminución del flujo de gas licuado desde $3.500 \mathrm{~L} / \mathrm{d}\left(0,219 \mathrm{~m}^{3} / \mathrm{h}\right)$ hasta $2.000 \mathrm{~L} / \mathrm{d}\left(0,125 \mathrm{~m}^{3} / \mathrm{h}\right)$ provoca una disminución en el calor cedido al medio por el horno, manteniendo constante el flujo de aire al horno, así como la temperatura de salida de los gases de combustión, desde $4,5 \times 10^{6} \mathrm{~kJ} / \mathrm{h}$ hasta $1,7 \times 10^{6} \mathrm{~kJ} / \mathrm{h}$, lo que equivale a una reducción de un 59,57\%. No obstante, las pérdidas generadas continúan siendo grandes representando el $31,66 \%$ del calor total que se evoluciona durante la combustión.

\section{ANÁLISIS ECONÓMICO}

Para llevar a cabo el análisis económico se determinó para cada alternativa la ganancia económica (GE), así como la relación beneficio costo $(\mathrm{B} / \mathrm{C})$ tomando como punto de partida los costos por concepto de materias primas y utilidades, así como los ingresos por concepto de detergente. Los resultados se muestran en la tabla 13. 
Tabla 13. Comparación económica de cada una de las alternativas

\begin{tabular}{|c|c|c|c|c|}
\hline Indicadores & $C B$ & $C E 1$ & $C E 2$ & $C E 3$ \\
\hline $\mathrm{GE}($ s/año $)$ & $-30.126,34$ & $-66.674,50$ & $49.611,28$ & $197.175,26$ \\
\hline$\Delta \mathrm{GE}(\$ / \mathrm{año})$ & - & $-36.548,16$ & $79.737,62$ & $227.301,60$ \\
\hline$\Delta \mathrm{GE}(\%)$ & - & $-321,32$ & 64,68 & 554,49 \\
\hline $\mathrm{B} / \mathrm{C}(\$ / \$)$ & 1,00 & 0,99 & 1,00 & 1,02 \\
\hline$\Delta \mathrm{B} / \mathrm{C}(\$ / \$)$ & - & 0,00 & 0,01 & 0,02 \\
\hline
\end{tabular}

Fuente: elaboración propia

Para profundizar en el análisis económico, en la alternativa de mayor ventaja, se realizó un análisis de sensibilidad de la ganancia ante la variación de los ingresos por venta de detergente y los egresos por concepto de ADBS e hidróxido de sodio. Para ello se definió como variable dependiente la ganancia y se consideraron cinco valores de cada variable independiente: el valor esperado $(0 \%)$, un valor optimista $(+5 \%)$, otro pesimista $(-5 \%)$ y valores extremos optimista $(+10 \%)$ y extremos pesimista $(-10 \%)$. Puede observarse en la figura 3, que la ganancia es sensible a todas las variables independientes analizadas, pero la mayor sensibilidad aparece ante la variable ingresos por venta de detergente que obtengan.

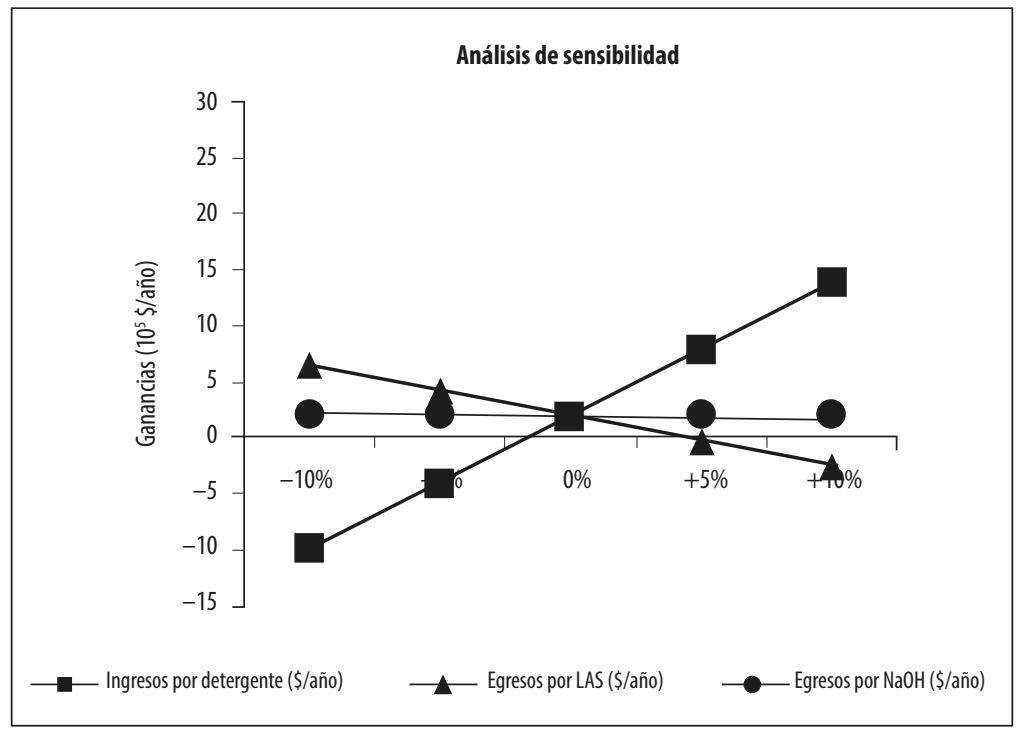

Figura 3. Resultados del análisis de sensibilidad.

Fuente: elaboración propia 


\section{CONCLUSIONES}

Se comprobó que el esquema de simulación desarrollado con el simulador de procesos químicos HYSYS arroja resultados que se corresponden con las condiciones reales de operación en el proceso de producción de detergentes en polvo, desde el punto de vista de los balances de materiales, lo que permite su uso para el análisis de alternativas de operación.

Por otro lado, los resultados obtenidos de los casos de estudio 1 y 2 muestran que es posible disminuir el $\mathrm{pH}$ de la pasta neutra en un 15,5\%, aproximadamente, aunque con la variante aplicada en el caso de estudio 2 se reduce la producción en un 4,2\%, mientras que se logra que el porcentaje de componente activo sea el exigido en la especificación. Lo anterior permite reajustar las condiciones de operación de modo que se eleve la productividad del proceso.

\section{REFERENCIAS}

[1] González, C. E. "Simulador de una columna de destilación binaria”. Tesis en opción del título de Ingeniero Industrial y en Sistemas Organizacionales, Escuela de Ingeniería, Universidad de La Salle. México DF. 2001.

[2] Hyprotech. "Hyprotech: simulation software for industry" Disponible en internet: http://www. ucalgary.ca/community/research/hyprotech. [Citado el 23 de febrero de 2013].

[3] Sardiñas, D. J. "Desarrollo de una base de conocimientos para el entrenamiento de los operarios y tecnólogos de la planta de detergentes de la Empresa Suchel Debón y propuesta de un sistema de control para el proceso de neutralización". [Tesis MSc], Instituto Superior Politécnico José Antonio Echeverría. 30 pp. 2012.

[4] Reglamento técnico de la empresa, Pto: 8226-01, Detergentes en polvo multiusos. Neutralización y batición. Proceso tecnológico operativo. Empresa Suchel Debón, 2013.

[5] Initial assessment report for 20th SIAM, UNEP Publications. Disponible en internet: jheinze@ johnadams.com [Citado el 25 de febrero de 2013].

[6] Adalid, G. N. "Diseño de una planta para la fabricación de detergente", Proyecto de fin de carrera, Universidad de Barcelona. 2001.

[7] Perry, H. R; Green, W. D. "Perry's Chemical Engineers' Handbook". McGraw-Hill. New York. Section 2. 2008.

[8] Sinnott, R. K. "Chemical Engineering Design”. Vol 6, 4th Edition. New Jersey. Elsevier. 1031pp. 2005.

[9] Carlson, E. C. "Don't gamble with physical properties for simulations". Chemical engineering progress, October pp35. 1996. 
[10] Aspentech. “Aspen HYSYS Simulation Basis” version 7. Aspen Technology, Inc. USA. 561 pp. 2008.

[11] Petrucci, R. H; Harwood, W. S. "General Chemistry: principles and modern applications". Prentice-Hall. New Jersey. 587 pp. 1999. 